\title{
Some Chromosomal Information in Haemonchus contortus Cobb, 1898
}

\author{
Manju Lata, A. K. Sharma and S. S. Lal \\ Department of Zoology, Institute of Advanced Studies, \\ Meerut University, Meerut, India
}

Accepted July 18, 1984

Some cytogenetical studies on the ovine and bovine strains of Haemonchus (wire worm) have been made by Bremner (1955) and Jambre (1979) to differentiate Haemonchus contortus and Haemonchus placei as the two distinct species both morphologically and cytologically. The present investigation has been made to study the chromosomes of Haemonchus contortus in which the sex chromosomes (XX) are not so large as in the case of Haemonchus placei.

\section{Material and method}

The mature male and female worms were collected from the intestine of sheep. Chromosomal preparation were made of the gonadal tissue of the worms by heat dry smear method. Gonads were first pretreated with hypotonic solution $(0.9 \%)$ of sodium citrate for 10-15 minutes and were later fixed in methanol-acetic acid $(3: 1)$, the tissue was then teased on a slide in $3-4$ drops $50 \%$ with the help of the fine needles, gently heated, dried, and, then stained with $2-4 \%$ buffered Giemsa.

\section{Observations}

Spermatogonial interphase nucleus (Fig. 1) shows positively heteropycnotic and round sex chromatin body which is found situated at the periphery of the nucleus. The premetaphase (Figs. 2,3) studied in more than 20 cells of different individuals invariably revealed 11 as the diploid chromosome number in the male and 12 in the female. All the chromosomes at premetaphase appeared as short elongated bodies. Karyogram (Fig. 4) prepared from spermatogonial premetaphase revealed the intergradation in the chromosome length, the largest pair is of $3 \mu \mathrm{m}$ while the smallest pair $1.7 \mu \mathrm{m}$. Single chromosome, at this stage, which shows positively heterochromatin is X-chromosome. The length of this chromosome is $1.7 \mu \mathrm{m}$. At telophase (Fig. 5) the identity of chromosomes is lost and two groups in the form of darkly stained masses were formed.

At pachytene (Fig. 6) all the thread like bivalents lie haphazardly at the plate. Counting of the chromosomes all along their length is not possible at this stage. Pachytene stage, studied in many cells, invariably showed at least one chromosome pair attached to the nucleolus.

Metaphase I (Fig. 7) revealed 5 highly condensed, dumb-bell shape bivalents and one univalent. This univalent again showed positive heteropycnosis and was 

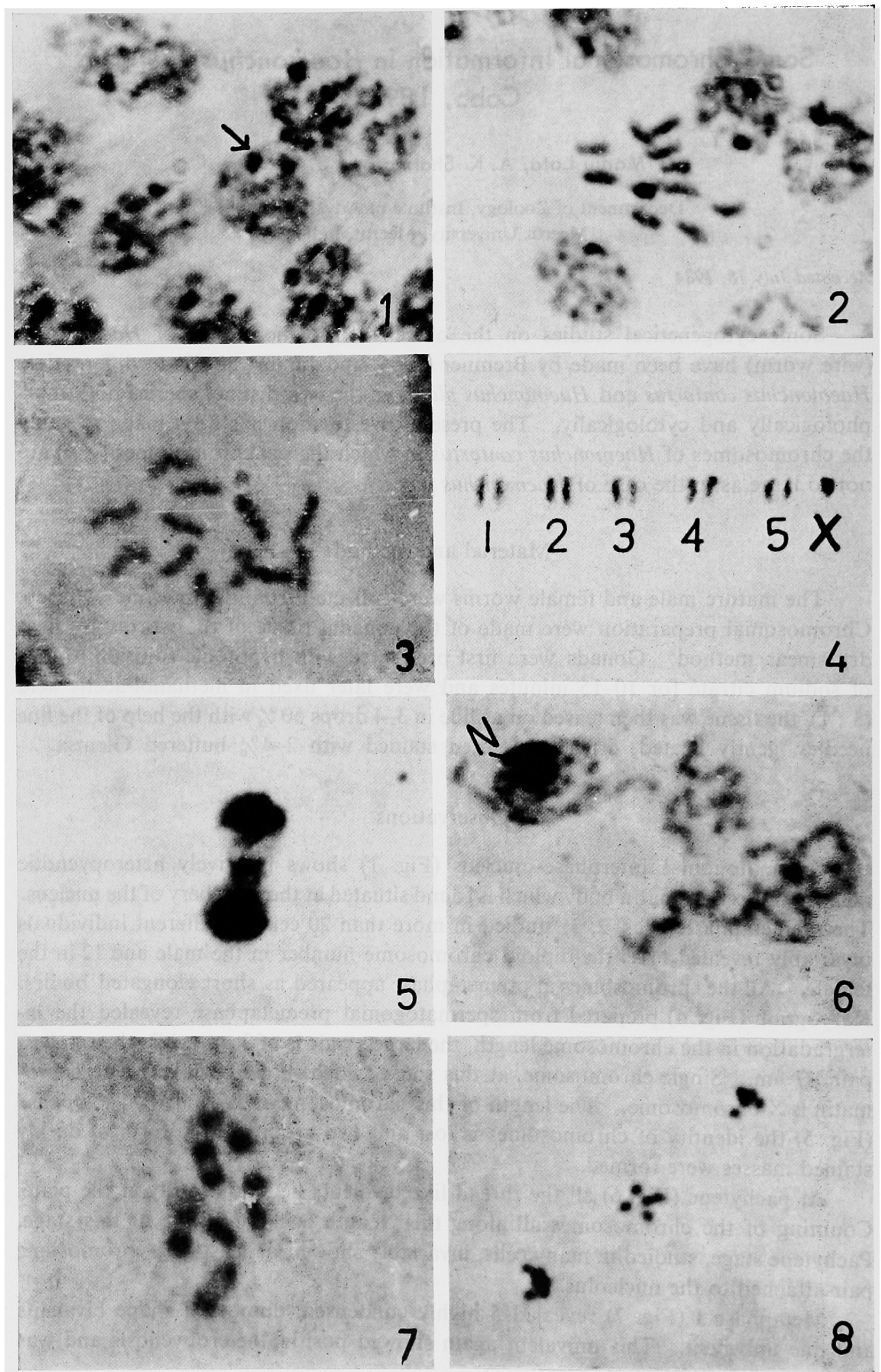
identified as the sex $(\mathrm{X})$ chromosome. At metaphase II (Fig. 8) two types of cells, some with 5 chromosomes another with 6 chromsomes were observed. At this stage all the elements appeared in the form of small dots without much variation in size.

\section{Discussion}

Haemonchus contortus belongs to the family Trichostrongylidae. So far only eight (8) species of nematode belonging to this family have been studied karyologically and all eight species invariably showed 6 as the haploid chromosome number. In the present species Haemonchus contortus the diploid chromosome number is 12 in the female and 11 in the male which confirmed the report by Bremner (1955) and Jambre (1979). In this nematode sex mechanism is of XO/XX type. Positively heteropycnotic sex chromatin body has also been observed in the interphase nucleus of the male sex. Sex chromosome $(\mathrm{X})$ was easily identifiable in the male during mitosis and meiosis both. Meosis is chiasmatic in both the male and the female. The presence of the sex chromatin body in the male has been a great controversy. If it is would be the $\mathrm{X}$ chromosome then it should also be in the female. Further researches are needed to say some thing confirm about this body.

\section{Summary}

The present investigation have been carried out on the chromosomes of Haemonchus contortus by heat dry smear technique. The diploid chromosome number in the male of this species is 11 while in the female it is 12 . The sex mechanism is of $\mathrm{XO} / \mathrm{XX}$ type. Heteropycnotic sex chromatin body has been observed at the periphery of interphase nucleus of the male. Meiosis shows that the male and the female both are chiasmatic. Sex chromosome $\mathrm{X}$ is easily distinguishable in the male sex due to the differential staining behaviour and an univalent nature.

\section{Acknowledgment}

The authors are thankful to Prof. K. C. Pandey, Head of the department, for providing laboratory facilities.

\section{References}

Bremner, K. C. 1955. Cytological polymorphism in the nematode Haemonchus contortus Cobb. 1898. Nature 174: 704-705.

Cobb, N. A. 1898. Extract from M.S. report on the parasites of stock. Agric. Gaz. N.S.W. 9: 296-321.

Jambre, Le. F. L. 1979. Hybridization studies of Haemonchus contortus (Rudolphi, 1803) and H. placei (Place, 1893). Inter. J. Parasitol. 9: 455-463.

Figs. 1-8. 1, interphase nucleus. Arrow indicates heteropycnotic sex chromatin body. 2, premetaphase (male.) 3, premetaphase (female). 4, karyogram prepared from Fig. 2. 5, telophase (male). 6, pachytene (N: nucleolus). 7, metaphase I (male). 8, metaphase II (male). 